mortality. It would simply mean that the cause of death would be different.

Enabling people to live longer-perhaps for 20 years after retirement-would create economic problems no less great than at present. There would perhaps be the compensation that more and more of the unemployed could be used in looking after the aged. Or will you be proposing what the Archbishop of Canterbury hinted at when financial resources really dry up ?

S EllisoN

London NW6

\section{A cautionary tale}

SIR,- 'Tis with a sense of grievous shock That one observed great Stephen Lock To perpetrate the awful sin Of leaving an unchecked reference in An article that bears his name. ${ }^{1}$ This blot on editorial fame Though "For Debate" is clear to see At least to Belloc and to me: It was not Lundy's noble dad Who did advise the tearful lad It was his grandpapa the Duke - Sir, what a liberty you took!

Edinburgh

JACK CORMACK

${ }^{1}$ Lock, S, British Medical fournal, 1976, 2, 1548.

* *Dr Cormack is right, but may I use some more lines of Belloc for my apology?

"When I am dead, I hope it may be said:

'His sins were scarlet, but his books were read.', -ED, $B M 7$.

\section{Management of Hodgkin's disease}

SIR,-Members of the British National Lymphoma Group will have been interested in the paper from St Bartholomew's Hospital on intensive investigation in the management of Hodgkin's disease (4 December, $p$ 1343). The results and conclusions in their paper are remarkably similar to those found in a much larger series of patients reported by the National Lymphoma Group last year. ${ }^{1}$

It is curious that the Barts authors made no mention of this paper in their own report.

SYLVIA WaTkINS

Lister Hospital,

${ }^{1}$ Report from British National Lymphoma Investigation, Clinical Radiology, 1975, 26, 151.

\section{Diffuse myelitis associated with rubella vaccination}

SIR,-The report by Dr S Holt and others ( 30 October, p 1037) is most interesting. My own observations on a similar case may be pertinent.

In April 1975 a 27-year-old married woman came under my care complaining of paraesthesiae of both legs with a mild paraparesis. She had increased reflexes in both legs with a left extensor plantar response and loss of pain and temperature sensation to $T 5$ bilaterally. There was decreased appreciation of vibration and position sense at both ankles. Eight days previously she had been vaccinated with live rubella virus. Her previous medical history and family history were non- contributory. She had an erythrocyte sedimentation rate (ESR) of $48 \mathrm{~mm}$ in the first hour and cerebrospinal fluid (CSF) examination showed clear fluid with $13 \times 10^{6}$ lymphocytes $/ 1\left(13 / \mathrm{mm}^{3}\right)$ and $0.42 \mathrm{~g}$ protein $/ 1$ with $10.4 \%$ gammaglobulin. The Wassermann reaction was negative. No virus could be isolated from plasma or CSF. A routine viral screen was entirely normal and a rubella antibody test showed a haemagglutination inhibition titre of $1 / 256$, with a complement fixation titre of less than 8 and a negative $\operatorname{IgM}$ sucrose density grading fractionation test. The patient was treated with prednisolone $80 \mathrm{mg}$ daily for three days, which was then reduced to $40 \mathrm{mg}$ for the following week. Over the next six weeks she made an excellent recovery.

She was seen again in the clinic six months later, this time complaining of pain on moving her left eye for the previous two weeks. She was found to have a left optic neuritis, decreased visual acuity of $6 / 60$ in the left eye compared with $6 / 12$ in the right, and a left large centrocaecal scotoma. The ESR on this occasion was normal and a lumbar puncture showed clear CSF without cells, protein content $0.52 \mathrm{~g} / 1$, and IgG $10.5 \%$. Attempts at vira isolation were negative and the rubella antibody titre was $1 / 256$. Routine tests for viral antibodies were normal. She was treated with intramuscular tetracosactrin $1 \mathrm{mg}$ daily for one week. She was seen again four months later when she had made an excellent recovery, the only abnormality being a little pallor of both optic discs.

Eight months after the second attack she had a further attack of paraesthesiae involving the two legs and on examination was found to have mild paraparesis, increased reflexes in both legs with bilateral extensor plantar responses, and pain and temperature sensory loss to T7 on the left and T5 on the right side. On this occasion she stated that her symptoms began one week after exposure to children with rubella. A lumbar puncture was not carried out. She was treated with a similar dose of tetracosactrin and made an excellent response once again over the ensuing six weeks. Viral studie on this occasion showed a rubella antibody titre of $1 / 128$, a complement fixation titre of less than 8 and no detectable antirubella $\mathrm{IgM}$. A routine vira antibody screen was negative. The patient has remained symptom-free since then.

This patient has a syndrome which seems indistinguishable from multiple sclerosis An attack has been precipitated twice by exposure to rubella virus, on the first occasion by vaccination and on the second by coming into contact with children who had rubella. Her illness responded to steroids. There were no abnormal findings apart from a raised ESR with a slight CSF pleocytosis in the first attack and a mildly raised CSF protein in the second. Failure to isolate the virus in no way militates against the disease being caused by a virus, though in virtually all cases of postinfectious encephalomyelitis no virus has been isolated. This finding has strengthened the hypothesis that post- or para-infectious encephalomyelitis represents a remote complication of infection -that is, immune-mediated demyelination. In support of this has been the demonstration of cell-mediated immunity to myelin encephalitogenic basic proteins in patients with acute disseminated encephalomyelitis. ${ }^{1}{ }^{2}$ How ever, in opposition to this view has been the isolation of measles virus ${ }^{3}$ and the demonstra tion of inclusion bodies in patients with post-measles encephalomyelitis. ${ }^{4}$ Postinfectious encephalomyelitis with post-mortem verification has previously been described following rubella, ${ }^{56}$ and indeed multiple sclerosis itself may occur following vaccination against several viral diseases. ${ }^{7}$ The immunopathological mechanisms by which involve ment of the nervous system occurs following vaccination is far from clear. It may well represent latent virus in cells which are attached by sensitised lymphocytes or humoral antibody or alternatively sensitisation to neural constituents.

Whether or not this patient suffers from relapsing acute disseminated encephalomyelitis or multiple sclerosis is a moot point and one that cannot be solved to date. There are points clinically in this case that favour both diagnoses and, indeed, pathologically verified cases have been described in which both diseases occured. ${ }^{8} 9$ The differences between the two diseases more likely result from genetic and immunological factors which govern the patient's reaction to the same agent. "Overlap" syndromes are therefore likely to occur. The further documentation of this association of diffuse demyelinating disorder with vaccination may help in our understanding of the cause of multiple sclerosis.

Peter O Behan

Institute of Neurological Sciences,

Southern General Hospital,

Glasgow

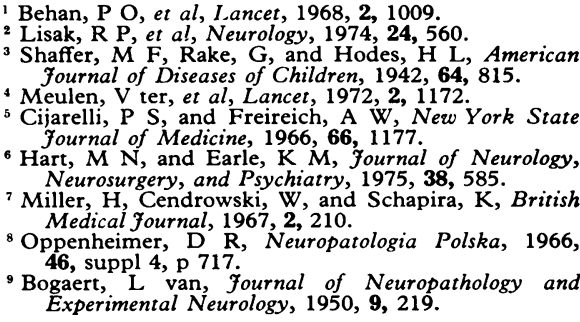

\section{Pancreatic diagnosis}

SIR,-In their recent comparison of pancreatic isotope scans, pancreatography, and Lundh tests Dr C J Mitchell and his colleagues (27 November, p 1307) found that a normal scan was strong evidence against pancreatic disease and recommended the scan as a screening test. In this unit with a particular interest in pancreatic diagnosis results and conclusions have been different. Our previous experience had been disappointing, but we set out here to make an assessment in collaboration with the department of nuclear medicine. Among 81 patients undergoing careful isotope scanning there were 30 with unequivocably normal pancreatic images. Twelve $(40 \%)$ proved to have definite pancreatic disease (two cancer, five severe chronic pancreatitis, five relapsing pancreatitis). Among 40 patients finally judged to have no pancreatic disease, only 16 had an unequivocally normal scan; 11 scans were abnormal and 13 equivocal. There were a total of 28 patients with unequivocally abnormal scans. Eleven (40\%) were finally judged to be free of pancreatic disease. Thus in this series scans had $40 \%$ false-positive and $40 \%$ false-negative results. We concluded that scans were of no clinical value and no longer use them.

There have been other recent developments. While computed tomography could not be regarded as a screening test, we have been impressed by the recent results of grey-scale ultrasonography, a technique which is quicker, cheaper, and presumably safer than isotope scanning.

However, at present when attempting the diagnosis of a patient who may have pancreatic disease we rely most heavily on clinical judgment and endoscopic pancreatography (ERCP). While the latter technique is complex and has potential hazards, these can be overcome. We have provided an adequate pancreatogram in $92 \%$ of our last 200 cases, with 